\title{
A 13-yr-old male with fever, malaise, body pains and dry cough
}

\author{
A.C. Defilippi*, P. Tomà ${ }^{\#}$, U.G. Rossi" , O. Sacco*, B. Ciravegna", C. Gambini' ${ }^{+}$, G.A. Rossi*
}

\section{Case history}

A previously healthy 13-yr-old male was evaluated at La Spezia Hospital, La Spezia, Italy, because of a 15-day history of fever, malaise, body aches and dry cough, which were not modified by treatment with amoxicillin.

A summary of the first admission blood tests is shown in table 1. The chest radiograph and the high-resolution computed tomography (HRCT) scans performed at that time are shown in figure $1 \mathrm{a}-\mathrm{c}$. Treatment with ampicillin, teicoplanin and netilmycin was administered for 7 days followed (as a result of no significant clinical response) by 12 days i.v. therapy with ciprofloxacine and imipenemcilastatine. The apyretic patient was discharged on oral ciprofloxacin. However, 2 weeks later, he was referred to the Giannina Gaslini Institute, Genoa, Italy, for further evaluations due to the recurrence of fever and dry cough.

On second admission, the patient's body temperature was $37.8^{\circ} \mathrm{C}$, his respiratory rate was 28 breaths $\cdot \mathrm{min}^{-1}$ and transcutaneous $\mathrm{O}_{2}$ saturation in room air was $98 \%$. Blood test results are shown in table 1. Chest radiograph and HRCT scans are shown in figure $2 \mathrm{a}-\mathrm{c}$. Fibreoptic bronchoscopy did not demonstrate any airway abnormality. Bronchoalveolar lavage (BAL) analysis showed as follows: 1) increased neutrophil percentage $(35 \%)$; b) no acid-fast bacilli, viruses, fungi or malignant cells; and 3) positive cultures for Streptococcus mitis and S. milleri. Microscopic examination of transbronchial-biopsy specimens obtained from the right lower lobe showed interstitial nonspecific lymphocyte infiltration. Treatment with ampicillin-tazobactam and vancomycin was prescribed for 10 days, with only slight improvement of the clinical and laboratory parameters but no further changes in the chest radiographs. A diagnostic surgical procedure was performed and specimens from the right lower lung lobe were sent to the pathologist for evaluation (fig. 3a-d).

Table 1.-Blood test results for the patient's first and second admission

\begin{tabular}{lcc}
\hline Investigations & $\begin{array}{c}1 \mathrm{st} \\
\text { admission }\end{array}$ & $\begin{array}{c}\text { 2nd } \\
\text { admission }\end{array}$ \\
\hline Haemoglobin $\mathrm{g} \cdot \mathrm{L}^{-1}$ & 88 & 105 \\
White cell counts particles $\cdot \mathrm{L}^{-1}$ & $12.6 \times 10^{9}$ & $22.5 \times 10^{9}$ \\
Platelet counts particles $\cdot \mathrm{L}^{-1}$ & $495 \times 10^{9}$ & 540 \\
Erythrocyte sedimentation & 90 & 105 \\
$\quad$ rate mm $1 \mathrm{st} \mathrm{h}^{-1}$ & & \\
C-reactive protein $\mathrm{g} \cdot \mathrm{L}^{-1}$ & 0.182 & 0.177 \\
Serum protein & Normal & \\
Immunoglobin electrophoresis & Normal & \\
Creatinine $\mu$ mol $\cdot \mathrm{L}^{-1}$ & 80 & \\
Complement component $\mathrm{C} 3 \mathrm{~g} \cdot \mathrm{L}^{-1}$ & 2.01 & \\
Complement component $\mathrm{C} 4 \mathrm{~g} \cdot \mathrm{L}^{-1}$ & 0.29 & \\
Antinuclear antibodies & Negative & Negative \\
Anti-neutrophil cytoplasmic antibodies & & Negative \\
Anti-streptolysin-O titre $\mathrm{UI} \cdot \mathrm{L}^{-1}$ & 5.790 & 2.859 \\
Neuron specific enolase & & Negative \\
Carcinoembryonic antigen & Negative \\
Alpha-foetoprotein & Negative & \\
Stains and culture of blood & Negative & \\
Pharyngeal aspirate & Negative & \\
Tuberculin skin test & Negative & \\
\hline
\end{tabular}

Data are presented as $n$ unless otherwise stated.
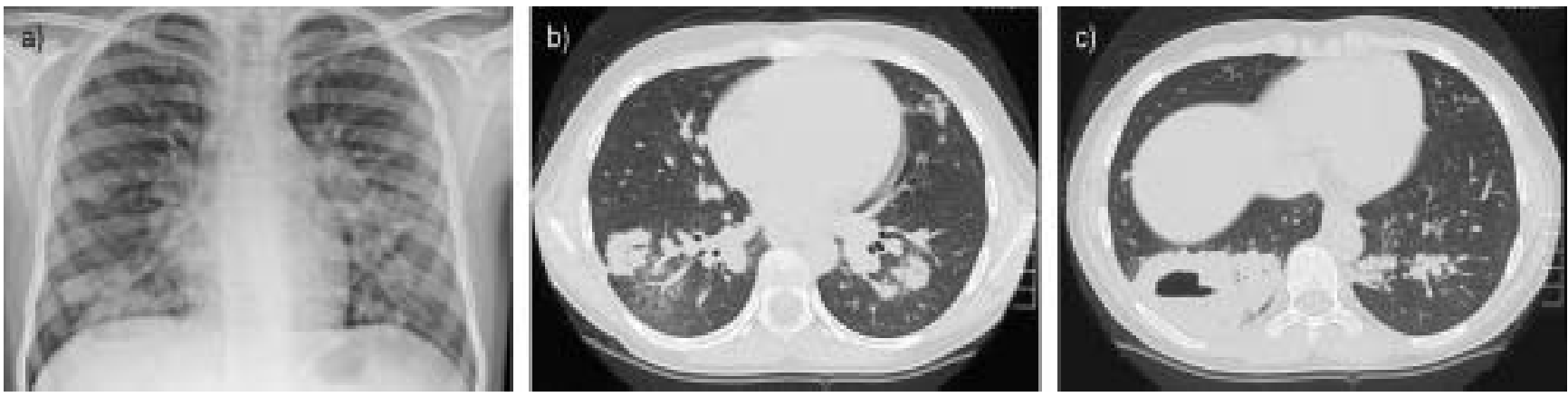

Fig. 1.-Chest radiograph (a) and high resolution computed tomography scans at two different levels (b and c) taken at the first admission.

\footnotetext{
*Pulmonary, ${ }^{\#}$ Radiology and ${ }^{+}$Pathology Units, and "Infectious Disease Chair, G. Gaslini Institute and University of Genoa, Genoa, Italy. Correspondence: G.A. Rossi, Pulmonary Disease Unit, G. Gaslini Institute, Largo G. Gaslini 5, 16147 Genoa, Italy. Fax: 39 010383953. E-mail: giovannirossi@ospedale-gaslini.ge.it
} 

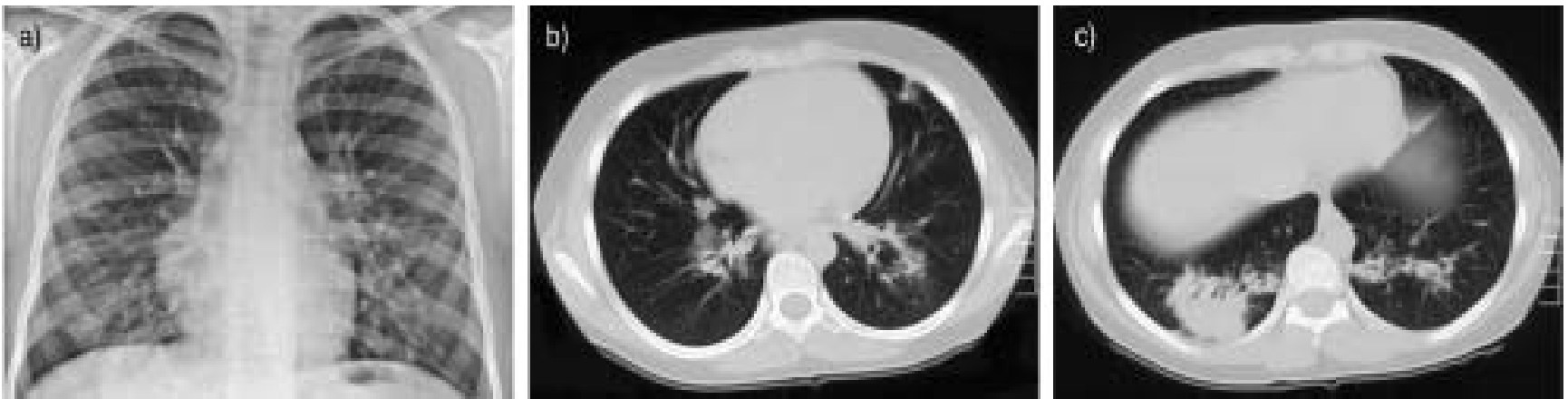

Fig. 2.-Chest radiograph (a) and high resolution computed tomography scans at two different levels (b and c) on admission to the Gaslini Institute 5 weeks later.
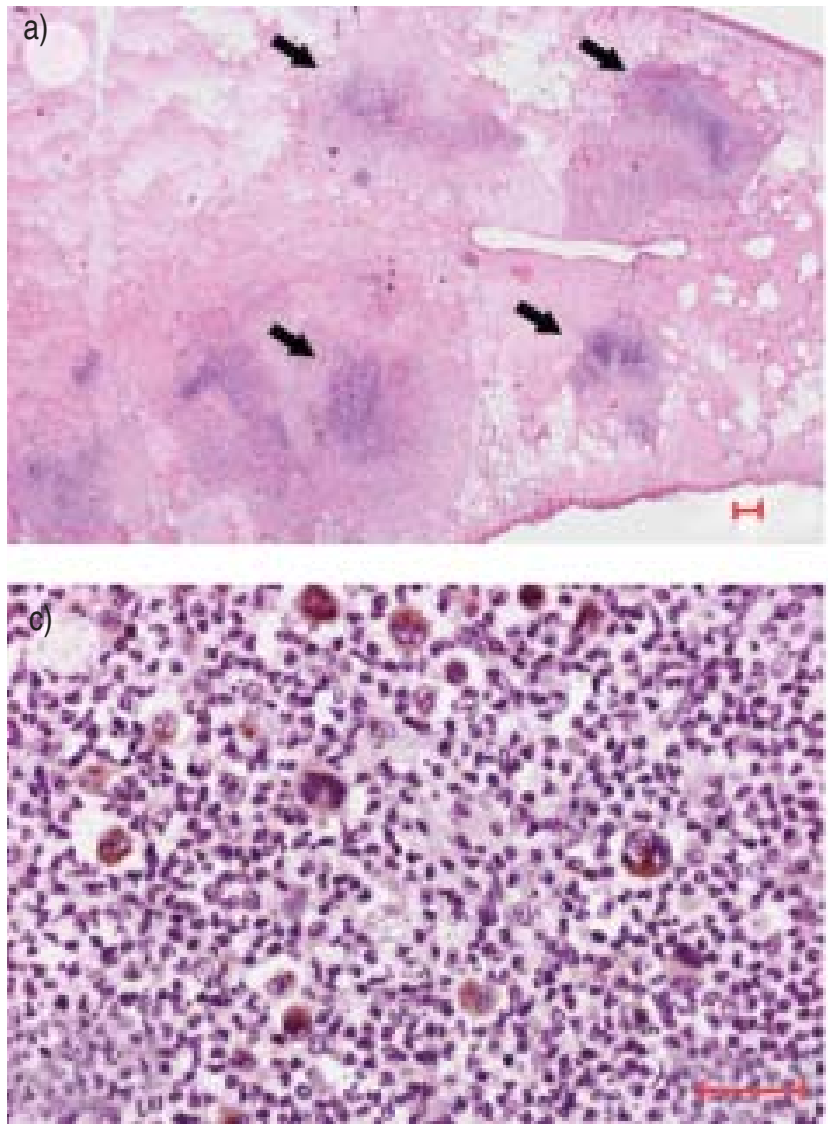
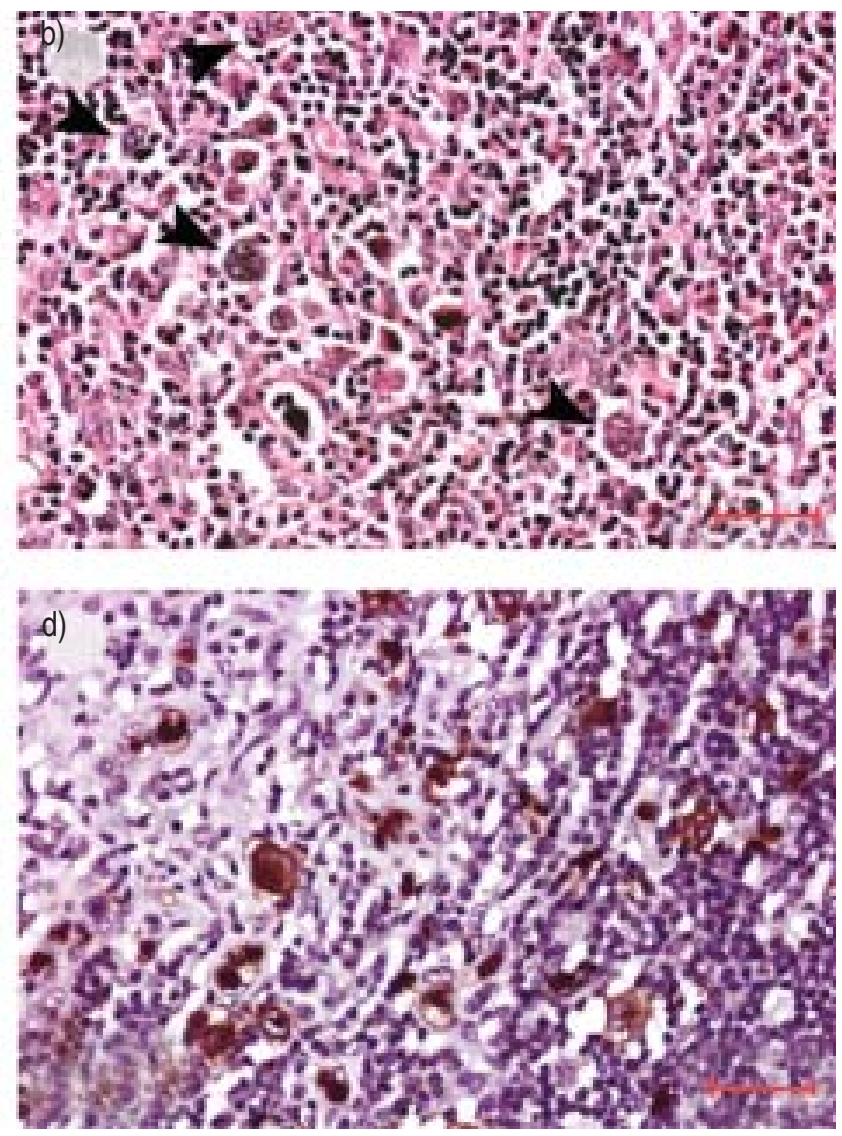

Fig. 3. - Histological preparations of lung biopsy specimen. a) Haematoxylin and eosin staining at lower magnification: internal scale bar=1mm b) Haematoxylin and eosin staining at higher magnification: internal scale bar $=50 \mu \mathrm{m}$. c) Immunostaining with the anti CD30 monoclonal antibody: internal scale bar $=50 \mu \mathrm{m}$. d) Immunostaining with the anti CD15 monoclonal antibody: internal scale bar=50 $\mu \mathrm{m}$.

PLEASE INTERPRET THE RADIOGRAPHS, COMPUTED TOMOGRAPHY SCANS AND LUNG BIOPSY RESULTS AND SUGGEST A DIAGNOSIS AND TREATMENT. 


\section{Interpretation}

\section{Chest radiography on the first admission}

Figure 1a shows bilateral, round-shaped, pulmonary infiltrates and bilateral hilar adenopathy.

\section{High-resolution computed tomography on the first admission}

HRCT of the chest depicts multiple bilateral nodular consolidations of different size, with air bronchograms, illdefined borders and hilar lymph node enlargement (fig. 1b). A larger space-occupying mass is detected in the right lower lobe with a thick-walled cavitation (fig. 1c).

\section{Chest radiography on the second admission}

Five weeks later, figure $2 \mathrm{a}$ shows partial regression of the pulmonary infiltrates and significant volume reduction of the hilar adenopathies.

\section{High-resolution computed tomography on the second admission}

HRCT of the chest better defines the significant regression of the bilateral nodular consolidations and the hilar lymph node enlargement (fig. 2b). A volume reduction of the stillpresent large space-occupying mass in the right lower lobe can be observed with no evidence of residual cavitation (fig. 2c).

\section{Pathology}

Figure 3 shows the histological evaluation of the resected pulmonary lobe specimens. At low magnification, multiple illdefined, centrally necrotic, nodular infiltrates can be detected in the lung parenchyma (fig. 3a). At a higher magnification, a nodule of lymphoid cells can be detected with large, multinucleated cells (arrows), with prominent inclusion-like nucleoli, perinucleolar halos and abundant eosinophilic cytoplasm, which are characteristic of the Reed-Sternberg cells. A background of small lymphocytes with several neutrophils and eosinophils was observed (fig. 3b). Immunoperoxidase staining demonstrated that the atypical cells showed reactivity for both CD30 (an activation antigen) and CD15 (a granulocyte-associated antigen), as is typical of classic Hodgkin's lymphoma (fig. 3c and d).

\section{Diagnostic considerations}

In the differential diagnosis of the present case, the current authors considered the following diagnoses: 1) infections: bacterial pneumonia, tuberculosis, non-tuberculosis mycobacterium infection, fungal infection; 2) malignancies: Ewing's sarcoma, osteosarcoma; 3) lymphoma: Hodgkin's and non-Hodgkin's; 4) idiopathic bronchiolitis obliterans with organising pneumonitis; and 5) vasculitis and rheumatic diseases.

\section{Surgical procedure and interpretation of surgical specimens}

A right-sided thoracotomy was performed and an infiltrated, sclerous right lower lobe was highlighted. A right lower lobectomy was then performed and the removed specimens were sent to the pathologist for morphologic evaluation of lung tissues.

Macroscopic features. In the resected lobe $(13 \times 9 \times 7 \mathrm{~cm})$, a white-greyish area $(4 \times 3 \mathrm{~cm})$ was detected with irregular margins. There was no evidence of bronchial lymphadenopathy.

Microscopic features. Biopsy specimen evaluation showed the pattern described previously for the interpretation of figure 3 , which was consistent with the diagnosis of nodular sclerosis Hodgkin's lymphoma (British National Lymphoma Investigation (BNLI) grade II) [1, 2].

Diagnosis: Classical Hodgkin's lymphoma, nodular sclerosis subtype NLI grade II, with probable associated multifocal $S$. mitis and $S$. milleri pneumonia

\section{Clinical course}

Staging procedures, including neck, thorax and abdomen CT scan, and skeletal and gallium pulmonary scintigraphy, demonstrated some bilateral pulmonary lesions and large hilar lymphadenopathy, but there was no evidence of neck and abdominal lymph node enlargement, hepato-splenomegaly or bone lesions. Complete haematological and biochemical screening showed only slight hypocromic anaemia. Chemotherapy chlorambucil-vincristine, procarbazine and prednisone/doxorubicin, bleomycin and vinblastine was prescribed, resulting in complete remission.

\section{Discussion}

Hodgkin's lymphoma is a malignancy that was first described in 1832; it has a bimodal distribution with regard to age, with an early peak occurring between ages 20-29 yrs, decreasing thereafter and reaching a second peak beyond the age of 50 yrs [2]. In children, this disorder accounts for approximately half of lymphomas, with an overall incidence rate of 14 per 100,000 under the age of 15 yrs. There is a slight male predominance in children $<10$ yrs, whereas it predominantly affects older adolescents females [2-4].

Hodgkin's lymphoma is classified into two categories: nodular lymphocyte-predominant Hodgkin's lymphoma and classical Hodgkin's lymphoma. The nodular lymphocytepredominant subtype, which constitutes $\sim 5 \%$ of all cases, is defined by the presence of a "nodular" background of small Bcells within a network of dendritic cells and of large neoplastic cells that express the B-cell-associated antigen CD20, but not CD15 or CD30 [1-3, 5]. The classic Hodgkin's lymphoma, which has four subtypes (i.e. nodular sclerosis, mixed cellularity, lymphocyte-rich and lymphocyte-depleted), is characterised by the presence of neoplastic cells that express CD15 and CD30 [1-3, 5]. In the developed world, the nodular sclerosis subtype is the most common of the four histological subtypes and is by far the most common type seen in adolescents and young adults [1-4].

Systemic symptoms associated with cough or dyspnoea are detectable in $\sim 25 \%$ of patients at presentation when intrathoracic involvement is present [2]. Indeed, approximately three-quarters of the patients, mostly those with the nodular sclerosis subtype, have mediastinal and/or hilar lymphadenopathy with or without lung parenchymal lesions in the early or advanced stages of the disease [6]. Rarely, Hodgkin's lymphoma of the lung is the only manifestation of the disease $[2,6,7]$. In a study performed in adults, 
pulmonary involvement was detected in $22 \%$ of the patients at some stage of their disease and a coexistent mediastinal lymphadenopathy was found in $>50 \%$ of the cases [8]. Nodules appear to be multiple in $86 \%$ and bilateral in $66 \%$ of cases, ranging 2-100 $\mathrm{mm}$ in size and irregularly marginated in $83 \%$ of patients $[6,7]$. When pulmonary manifestations are present at initial diagnosis, as in the current authors' patient, they are always associated with hilar or mediastinal lymphadenopathy: indeed, the typical appearance of pulmonary Hodgkin's lymphoma consisted of multiple, irregularly marginated pulmonary nodules [6-8]. Cavitation of nodules occurs in $<1 \%$ of patients and may develop initially or after treatment [6-9]. Little is known about the incidence and the characteristics of pulmonary lesions in Hodgkin's lymphoma in children [6-10].

Therefore, in the current case, the entire clinical picture (i.e. multiple pulmonary nodules and intermittent fever) could have been related only to Hodgkin's lymphoma. However, since virtually all the pulmonary lesions disappeared or were reduced in size after antibiotic treatment and two different pathogens ( $S$. milleri and $S$. mitis) were detected by BAL, the hypothesis of an associated streptococcal infection should be considered. $S$. milleri has recently emerged as an important pathogen involved in the aetiology of community-acquired pneumonia and of acute respiratory tract infections in patients with moderate to severe underlying diseases, whereas $S$. mitis has also been detected as causative agent of bacteraemic pneumonia in children [11, 12]. Interestingly, a recent communication showed regression of nodular sclerotic type Hodgkin's lymphoma lung nodules in a 33-yr-old female by prolonged treatment with ciprofloxacin and clarithromycin, suggesting the hypothesis that a bacterial infection could also play a role in the pathogenesis of Hodgkin's lymphoma, as demonstrated for other malignant and nonmalignant neoplasms [13].

The relative, incomplete efficacy of the various antibiotic treatments in the current authors' present case may be related to the ability of $S$. milleri and $S$. mitis to develop antibiotic resistance, or to the presence of a defective anti-infectious immune response related to the underlying lymphoma. For many years, it has been unclear whether "Hodgkin's disease" was a neoplasm or an immunological or infectious disease. Recently, it has been clearly shown that Reed-Sternberg cells are monoclonal B-cells with rearranged and somatically mutated immunoglobulin genes [14, 15], whereas several epidemiological and experimental studies have highlighted the fact that Hodgkin's lymphoma is often associated with an infectious agent: the Epstein-Barr virus (EBV) [2, 4]. Association with the EBV varies with age; childhood Hodgkin's lymphoma is almost invariably associated, whereas the lowest rates of EBV-associated Hodgkin's lymphoma are reported in adolescents and in young adults (age: 15-34 yrs), raising the possibility that alternative lymphotropic viruses could possibly be implicated in the pathogenesis $[2,4]$. The combination of the monoclonal expansion of B-cells and latent viral infection may explain why Hodgkin's lymphoma may be associated with defects in cellular immunity, facilitating opportunistic and nonopportunistic infections, and lowering the efficacy of antibiotic treatments.

The hypothesis that bacterial infections, besides being associated to Hodgkin's lymphoma, may also play a role in the pathogenesis of this lymphoma deserves further investigations.

\section{References}

1. Leclair SJ, Rodak BF. The new WHO nomenclature: lymphoid neoplasms. Clin Lab Sci 2002; 15: 55-59.

2. Yung L, Linch D. Hodgkin's lymphoma. Lancet 2003; 361: 943-951.

3. Hansmann ML, Willenbrock K. WHO classification of Hodgkin's lymphoma and its molecular pathological relevance. Pathologe 2002; 23: 207-218.

4. $\quad$ Potter R. Paediatric Hodgkin's disease. Eur J Cancer 1999; 35: 1466-1476.

5. Torlakovic E, Torlakovic G. B-cell markers in lymphocyte predominance Hodgkin disease. Arch Pathol Lab Med 2002; 126: 862-863.

6. Diederich S, Link TM, Zuhlsdorf H, Steinmeyer E, Wormanns D, Heindel W. Pulmonary manifestations of Hodgkin's disease: radiographic and CT findings. Eur Radiol 2001; 11: 2295-2305.

7. Grimwade DJ, Chopra R, King A, et al. Detection and significance of pulmonary Hodgkin's disease at autologous bone marrow transplantation. Bone Marrow Transplant 1994; 13: 173-179.

8. Catterall JR, McCabe RE, Brooks RG, Remington JS. Open lung biopsy in patients with Hodgkin's disease and pulmonary infiltrates. Am Rev Respir Dis 1989; 139: 1274-1279.

9. Horak E, Olinsky A, Chow CW, Waters K, Sawyer SM. Multiple cavitating pulmonary nodules and clubbing in a 12year-old girl. Pediatr Pulmonol 2002; 34: 147-149.

10. Atra A, Higgs E, Capra M, et al. Isolated parenchymal lung involvement in children with stage IV Hodgkin's disease: results of the UKCCSG HD8201 and HD9201 studies. $\mathrm{Br}$ J Haematol 2002; 119: 441-444.

11. Porta G, Rodriguez-Carballeira M, Gomez L, et al. Thoracic infection caused by Streptococcus milleri. Eur Respir J 1998; 12: $357-362$.

12. Gaudreau C, Delage G, Rousseau D, Cantor ED. Bacteremia caused by viridans streptococci in 71 children. Can Med Assoc J 1981; 125: 1246-1249.

13. Sauter C, Blum S. Regression of lung lesions in Hodgkin's disease by antibiotics: case report and hypothesis on the etiology of Hodgkin's disease. Am J Clin Oncol 2003; 26: $92-$ 94.

14. Marafioti T, Hummel M, Anagnostopoulos I, et al. Origin of nodular lymphocyte-predominant Hodgkin's disease from a clonal expansion of highly mutated germinal-center B cells. $N$ Engl J Med 1997; 337: 453-458.

15. Kanzler H, Kuppers R, Hansmann ML, et al. Hodgkin and Reed-Sternberg cells in Hodgkin's disease represent the outgrowth of a dominant tumor clone derived from (crippled) germinal center B cells. J Exp Med 1996; 184: $1495-1505$. 